\title{
Exoplanet Discoveries with the CoRoT Space Observatory ${ }^{1}$
}

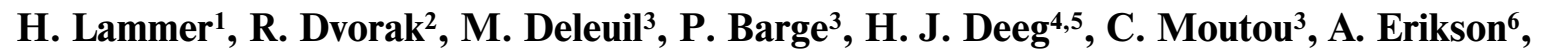
Sz. Csizmadia $^{6}$, B. Tingley, ${ }^{4,5}$ H. Bruntt ${ }^{7}$, M. Havel $^{8}$, S. Aigrain ${ }^{9,10}$, J. M. Almenara ${ }^{4,5}$, R. Alonso ${ }^{11}$, M. Auvergne ${ }^{7}$, A. Baglin 7 , M. Barbieri ${ }^{3,12}$, W. Benz ${ }^{13}$, A. S. Bonomo ${ }^{3}$, P. Bordé ${ }^{14}$, F. Bouchy ${ }^{15,16}$, J. Cabrera ${ }^{6,17}$, L. Carone $^{18}$, S. Carpano ${ }^{19}$, D. Ciardi' ${ }^{20}$, S. Ferraz-Mello ${ }^{21}$, M. Fridlund ${ }^{19}$, D. Gandolfi22, J.-C. Gazzano ${ }^{3}$, M. Gillon ${ }^{23}$, P. Gondoin ${ }^{19}$, E. Guenther ${ }^{22}$, T. Guillot ${ }^{8}$, R. den Hartog ${ }^{19}$, J. Hasiba ${ }^{1}$, A. Hatzes ${ }^{22}$, M. Hidas ${ }^{24,25}$, G. Hébrard ${ }^{15}$, L. Jorda ${ }^{3}$, P. Kabath ${ }^{6}$, A. Léger $^{14}$, T. Lister ${ }^{24}$, A. Llebaria ${ }^{3}$, C. Lovis ${ }^{21}$, M. Mayor ${ }^{11}$, T. Mazeh $^{26}$, A. Mura ${ }^{27}$, M. Ollivier ${ }^{14}$, H. Ottacher ${ }^{1}$, M. Pätzold ${ }^{18}$, F. Pepe ${ }^{11}$, F. Pont ${ }^{9}$, D. Queloz ${ }^{11}$, M. Rabus ${ }^{4,5}$, H. Rauer 6 ,28, D. Rouan ${ }^{7}$, B. Samuel ${ }^{14}$, J. Schneider ${ }^{17}$, A. Shporer ${ }^{26}$, B. Stecklum ${ }^{22}$, M. Steller ${ }^{1}$, R. Street ${ }^{24}$, S. Udry ${ }^{11}$, J. Weingrill ${ }^{1}$, and G. Wuchterl ${ }^{22}$

${ }^{1}$ Space Research Institute, Austrian Academy of Science, Schmiedlstrasse 6, A-8042 Graz, Austria

${ }^{2}$ University of Vienna, Institute of Astronomy, Turkenschanzstrasse 17, A-1180 Vienna, Austria

${ }^{3}$ Laboratoire d'Astrophysique de Marseille, CNRS and Université de Provence, F-13388 Marseille, France

${ }^{4}$ Instituto de Astrofisica de Canarias, C. Via Lactea S/N, E-38205 La Laguna, Tenerife, Spain

${ }^{5}$ Department de Astrofisica, Universidad de La Laguna, E-38200, Tenerife, Spain

${ }^{6}$ Institute of Planetary Research, German Aerospace Center, Rutherfordstrasse 2, D-12489 Berlin, Germany 5_LESIA

${ }^{7}$ Observatoire de Paris, CNRS, Place J. Janssen, 92195 Meudon cedex, France

${ }^{8}$ Université de Nice-Sophia Antipolis, Observatoire de la Cóte d'Azur, CNRS UMR 6202, France

${ }^{9}$ School of Physics, University of Exeter, Exeter, EX4 4QL, UK

${ }^{10}$ Oxford Astrophysics, University of Oxford, Keble Road, Oxford OX1 3RH, UK

${ }^{11}$ Observatoire de l'Université de Genéve, 51 chemin des Maillettes, CH 1290 Sauverny, Switzerland

${ }^{12}$ Dipartimento di Astronomia, Universitá di Padova, 35122 Padova, Italy

${ }^{13}$ Universitat Bern, Physics Institute, Sidlerstrasse 5, CH 3012 Bern, Switzerland

${ }^{14}$ Institut d'Astrophysique Spatiale, Université Paris XI, F-91405 Orsay, France

${ }^{15}$ IAP, 98 bis boulevard Arago, F-75014 Paris, France

${ }^{16}$ Observatoire de Haute-Provence, CNRS/OAMP, 04870 St Michel l'Observatoire, France

${ }^{17}$ LUTH, Observatoire de Paris, CNRS and Université Paris Diderot, F-92195 Meudon, France

${ }^{18}$ Rheinisches Institut für Umweltforschung an der Universitát zu Kóln, D-50931, Kóln, Germany

${ }^{19}$ Research and Scientific Support Department, ESTEC/ESA, 2200 AG Noordwijk, The Netherlands

${ }^{20}$ NASA Exoplanet Science Institute/Caltech, South Wilson Avenue, Pasadena, California 91125, USA

${ }^{21}$ Institute of Astronomy, Geophysics and Atmospheric Sciences, Universidade de Saó Paulo, Brasil

${ }^{22}$ Thuringer Landessternwarte, Sternwarte 5, D-07778 Tautenburg, Germany

${ }^{23}$ University of Liége, Sart Tilman, Liége 1, Belgium

${ }^{24}$ Las Cumbres Observatory Global Telescope Network, Inc., Santa Barbara, California 93117, USA

${ }^{25}$ Sydney Institute for Astronomy, School of Physics, The University of Sydney, Australia

${ }^{26}$ School of Physics and Astronomy, Raymond and Beverly Sackler Faculty of Exact Sciences, Tel Aviv University, Tel Aviv 69978, Israel

${ }^{27}$ Instituto di Fisica dello Spacio Interplanetario-CNR, Rome, Italy

${ }^{28}$ Center for Astronomy and Astrophysics, TU Berlin, Hardenbergstrasse 36, 10623 Berlin, Germany

Received April 19, 2010

\footnotetext{
${ }^{1}$ The article is published in the original.
} 


\begin{abstract}
The CoRoT space observatory is a project which is led by the French space agency CNES and leading space research institutes in Austria, Brazil, Belgium, Germany and Spain and also the European Space Agency ESA. CoRoT observed since its launch in December 27, 2006 about 100000 stars for the exoplanet channel, during 150 days uninterrupted high-precision photometry. Since the The CoRoT-team has several exoplanet candidates which are currently analyzed under its study, we report here the discoveries of nine exoplanets which were observed by CoRoT. Discovered exoplanets such as CoRoT-3b populate the brown dwarf desert and close the gap of measured physical properties between usual gas giants and very low mass stars. CoRoT discoveries extended the known range of planet masses down to about 4.8 Earth-masses (CoRoT-7b) and up to 21 Jupiter masses (CoRoT-3b), the radii to about $1.68 \times 0.09 R_{\text {Earth }}\left(\right.$ CoRoT-7b) and up to the most inflated hot Jupiter with $1.49 \times 0.09 R_{\text {Earth }}$ found so far (CoRoT-1b), and the transiting exoplanet with the longest period of 95.274 days (CoRoT-9b). Giant exoplanets have been detected at low metallicity, rapidly rotating and active, spotted stars. Two CoRoT planets have host stars with the lowest content of heavy elements known to show a transit hinting towards a different planethost-star-metallicity relation then the one found by radial-velocity search programs. Finally the properties of the CoRoT-7b prove that rocky planets with a density close to Earth exist outside the Solar System. Finally the detection of the secondary transit of CoRoT- $1 \mathrm{~b}$ at a sensitivity level of $10^{-5}$ and the very clear detection of the "superEarth" CoRoT-7b at $3.5 \times 10^{-4}$ relative flux are promising evidence that the space observatory is being able to detect even smaller exoplanets with the size of the Earth.
\end{abstract}

DOI: $10.1134 / \mathrm{S} 0038094610060055$

\section{INTRODUCTION}

The CoRoT (Convection, Rotation and planetary Transits) space observatory is a project which is led by the French space agency CNES and different European countries, leading space research institutes in Austria, Brazil, Belgium, Germany and Spain and the European Space Agency ESA. Before the discovery of the first exosolar gas giant in 1995, the CoRoT project focused on Asteroseismology of variable stars. After the discovery of the first hot Jupiter 51 Peg, the search for exoplanets was included in the CoRoT-program (Schneider and Chevreton, 1990). The CoRoT satellite is $4 \mathrm{~m}$ long, a mean diameter of $2 \mathrm{~m}$ and has a mass of $630 \mathrm{~kg}$. The accuracy of the pointing is $0.5 \mathrm{arcsec}$ with a capacity of the telemetry of $1.5 \mathrm{Gbit} /$ day. There are three systems which are operating on the satellite: an afocal telescope with 2 parabolic mirrors and aperture of $27 \mathrm{~cm}$ a cylindric baffle $2 \mathrm{~m}$ long (CoRoTel), a wide field camera consisting of a dioptric objective of 6 lenses where the focal unit is equipped with 4 frametransfer-CCD $2048 \times 4096$ (CoRoTcam), and four CCDs are for the exoplanet program and two are for the seismology program. CoRoTcase is hosting the electronics and the software managing the aperture photometry processing.

For the exoplanet CCD the total number of stars with magnitudes between 11 and 16 is about 12000 and the flux is measured every $512 \mathrm{~s}$ consisting of 16 individual exposures of $32 \mathrm{~s}$. A bi-prism in the focal block dedicated to exoplanets allows getting chromatic information in red, green and blue for brighter stars, but for faint stars only the standard one band white photometry is performed (Dvorak et al., 2010). The polar orbit of the satellite allows observing 150 days in the long run, and between 20 and 30 days in the short run, both in the direction of the center respectively anti-center of the galaxy, which makes two reversal maneuvers necessary per year.

The satellite was successfully launched on December, 27th 2006 from Baikonour Kosmodrome. In orbit
CoRoT started its observations in February 2007. The satellite life-time was initially scheduled for 3 years of observations but recently the extension for another three years was approved. Some of the results obtained with measurements of the CoRoT mission were published in a whole volume of the journal Astronomy and Astrophysics in 2009 (Baglin et al., 2009). After exoplanet candidates are discovered due to the light curve analysis, time consuming radial velocity (RV) measurements from ground-based telescopes (HARPS spectrograph in La Silla, $3.6 \mathrm{~m}$ telescope; SOPHIE spectrograph in the Observatoire de Haute de Provence; Coudé echelle spectrograph from the $2 \mathrm{~m}$ telescope, Tautenburg (TLS); UVES and FLAMES spectrographs in Chile; spectrographs at McDonald observatory, Provence (1.93 $\mathrm{m}$ telescope) and the CORALIE spectrograph on the 1.2 Swiss telescope in La Silla) have to be performed to confirm real planet candidates. At present time (April 2010), 12 exoplanets have been discovered by CoRoT and confirmed via ground-based RV follow-up campaigns. But many more planet candidates are in the analysis pipeline and wait for confirmation by follow-up campaigns. In the following Section 2 we will describe the first discovered nine CoRoT planets and in Section 3 we will discuss some preliminary characterization of the discovered exoplanets, with a focus on the first transiting "super-Earth" CoRoT-7b.

\section{THE FIRST NINE CONFIRMED EXOPLANETS DISCOVERED BY COROT}

CoRoT-1b (Barge et al., 2007): The exoplanet discovered by CoRoT is a Jupiter-mass gas giant $\left(1.03 M_{\mathrm{Jup}}\right)$ with a surprisingly large radii of $1.49 R_{\mathrm{Jup}}$ in front of a G0V star. Due to the inflated size the planet has a very small density $\left(0.38 \mathrm{~g} \mathrm{~cm}^{-3}\right)$ compared to all other planets found up to now. The light curve analysis of CoRoT- $1 \mathrm{~b}$ gave a very well determined period of 1.5 days because 34 transits could be used for its com- 


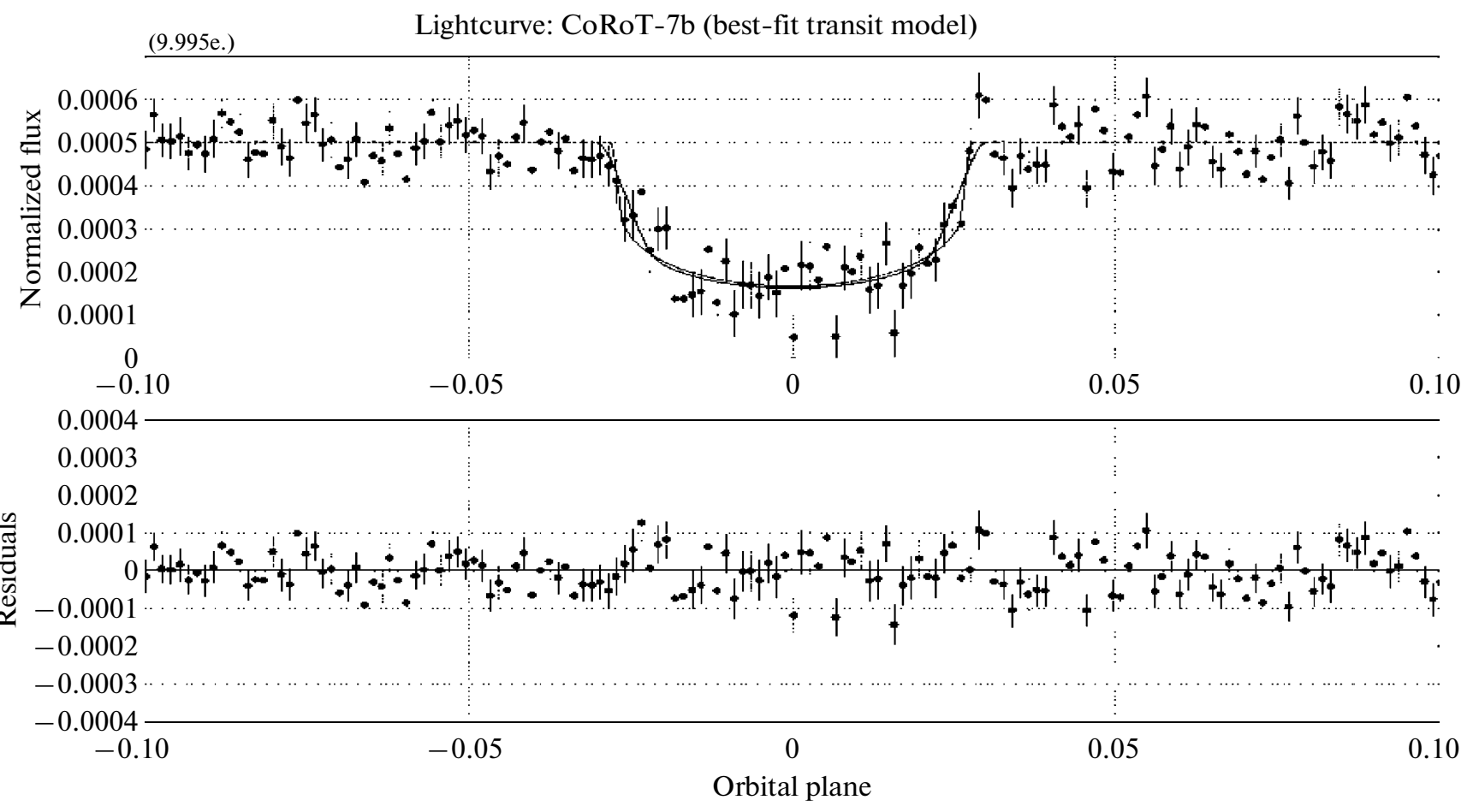

Fig. 1. Normalized and phase folded lightcurves of 153 transits of the first discovered transiting super-Earth, CoRoT-7b (after Léger et al., 2009).

putation. RV follow-up observations were undertaken with the SOPHIE spectrograph for 9 different positions of the planet so that its nature could be confirmed.

CoRoT-2b (Alonso et al., 2008): The second confirmed planet of CoRoT was derived by analyzing 78 transits during the 150 days of observations of CoRoT$2 \mathrm{~b}$ and after removing the signal of the star's rotation and activity, which is due to large spots on its surface. The planet orbits a K0V star with a metallicity of $[\mathrm{Fe} / \mathrm{H}]=0$ in 1.74 days. The ground-based RV followup indicates that the planet has a mass of $3.3 M_{\text {Jup }}$ and a radius of $1.465 R_{\mathrm{Jup}}$. With the use of very precise RV follow-up observations with the CORALIE, HARPS and SOPHIE instruments it was possible to determine an inclination of $\lambda=7.2^{\circ}$ between the planetary orbit and the equator of the central star.

CoRoT-3b (Deleuil et al., 2008): The third CoRoT planet was discovered by analyzing 34 transits from 152 days of observations. CoRot- $3 b$ orbits around a F3V-type star with a radius of $1.56 M_{\text {Sun }}$ with a period of 4.26 days. The results were a big surprise because with a size comparable to that of Jupiter, RV follow-up observations by HARPS, SOPHIE, CORALIE and TLS and measurements from Mc-Donald observatory indicated that the mass of the planet could be determined as 21.66 as heavy than Jupiter, which clearly distinguishes this planet from the normal close-in gas giants. This discovery indicates that CoRoT-3b is either a brown dwarf or a member of a new class of planets.

CoRoT-4b (Aigrain et al., 2008; Moutou et al., 2008): CoRoT-4b is also a gas giant which has a deflated size of about $1.2 R_{\text {Jup }}$. RV measurements with HARPS and SOPHIE confirmed its planetary nature and yield a mass of $0.71 M_{\text {Jup }}$. The planet orbits on a circular orbit around a young FOV-type star with a period of 9.2 days. Due to its large size and small mass the planet's mean density is about $0.52 \mathrm{~g} \mathrm{~cm}^{3}$. Furthermore, a detailed analysis of the light curve shows that the host star has an active surface with rapidly evolving starspots. From this analysis it was possible to derive a rotation period of the star of about 8.87 days, which is within its error bars consistent with the period of the planet.

CoRoT-5b (Rauer et al., 2009): After an analysis of 27 transits and an observation time of 112 days CoRoT-5b was discovered. The light curve is shown in Fig. 1? with a decrease in flux of $1.5 \%$. The light curve analysis led to an orbital period of 4 days. CoRoT-5b is also a very strange planet with a large radius of $1.4 R_{\text {Jup }}$ and a very low mass of about $0.47 M_{\text {Jup }}$. The mean density of CoRoT-5b is therefore one of the lowest for known gas giants of about $0.217 \mathrm{~g} \mathrm{~cm}^{-3}$. The host star of CoRoT-5b is a relatively old F9V-type star with an age of about 5.5-8.3 Gyrs star with the mass of the Sun and a slightly larger radius. By extensive ground-based RV follow-up observations with 
Physical parameters of nine exoplanets which were discovered by the CoRoT space observatory (e.g. Dvorak et al., 2010; Bordé et al., 2010; Deeg et al., 2010) Thermal mass loss rates $L_{\text {th }}$ are correspond to the estimations of Lammer et al. (2009) and heating efficiencies between $10-25 \%$

\begin{tabular}{c|c|c|c|c|c|c|c|c}
\hline Planet & Period, $\mathrm{d}$ & $a, \mathrm{AU}$ & inc., deg & ecc. & $\begin{array}{c}\text { Mass, } \\
M / M_{\text {Jup }}\end{array}$ & $\begin{array}{c}\text { Radius, } \\
R / R_{\text {Jup }}\end{array}$ & $\begin{array}{c}\text { Density, } \\
\mathrm{g}^{\mathrm{dm}^{-3}}\end{array}$ & $L_{\mathrm{th}}, \%$ \\
\hline CoRoT-1b & 1.50 & 0.0254 & 85.10 & 0 & 1.03 & 1.49 & 0.38 & $1.3-3.07$ \\
CoRoT-2b & 1.74 & 0.0281 & 87.84 & 0 & 3.31 & 1.465 & 1.31 & $0.06-0.15$ \\
CoRoT-3b & 4.25 & 0.0570 & 85.90 & 0 & 21.66 & 1.01 & 26.4 & - \\
CoRoT-4b & 9.20 & 0.0900 & 90.00 & $\sim 0$ & 0.72 & 1.19 & 0.525 & $0.12-0.3$ \\
CoRoT-5b & 4.03 & 0.0494 & 85.83 & 0.09 & 0.467 & 1.388 & 0.217 & $1.56-3.75$ \\
CoRoT-6b & 8.88 & 0.0855 & 89.07 & $<0.1$ & 2.96 & 1.166 & 1.94 & $\leq 1$ \\
CoRoT-7b & 0.85 & 0.0172 & - & 0 & 0.015 & 0.172 & 4.23 & - \\
CoRoT-8b & 6.21 & 0.0630 & 88.40 & 0 & 0.22 & 0.57 & 1.6 & $0.036-0.1$ \\
CoRoT-9b & 95.27 & 0.3600 & 89.99 & 0.11 & 0.84 & 1.05 & 0.9 & - \\
\hline
\end{tabular}

HARPS a very low metallicity ratio $[\mathrm{M} / \mathrm{H}]=-0.25$ could be determined.

CoRoT-6b (Fridlund et al., 2010): The 6th exoplanet discovered has an orbital period of 8.89 days, is about three times more massive and has a size which is about $15 \%$ larger than Jupiter in the Solar System. This mass-radius relation yields a high density of about $1.94 \mathrm{~g} \mathrm{~cm}^{-3}$. Due to a detailed analysis of RV measurements and photometric observations led to a full characterization of the star-planet system indicating that the planets host star with approximately the mass of the Sun contains a surprisingly low metallicity of $[\mathrm{Fe} / \mathrm{H}=-0.2]$.

CoRoT-7b (Léger et al., 2009): The most important observation by the CoRoT space observatory so far is the discovery of the size of the first "superEarth"-type exoplanet whose normalized and phasefolded lightcurve of 153 transits is shown in Fig. 1. The CoRoT-7b's radius was determined via photometric and spectroscopic measurements and turned out to be slightly smaller than 2 times the Earth's radius $\left(R_{\mathrm{pl}}=\right.$ $\left.1.78 R_{\text {Earth }}\right)$. Detailed ground-based RV follow-up with HARPS resulted in a mass of this planet corresponding to about 4.8 $M_{\text {Earth }}$ (Queloz et al., 2009) and in a mean density of about $5.6 \mathrm{~g} \mathrm{~cm}^{-3}$ which is similar to that of the Earth. This first fully characterize "superEarth" orbits at a very close-distance of about $0.017 \mathrm{AU}$ around a G9V-type solar like star with a metallic of 0.03 . After an analysis of 153 transits it turned out that CoRoT-7b orbit in a circular orbit at a distance of only approximately 4 times the radius of its host star. CoRoT-7b is the first rocky planet which moves in only 0.854 days around the relatively young main sequence star which has a rotation period of 23 days.

Due to the extensive RV follow-up observations Queloz et al. (2009) discovered a second exoplanet in the same system which orbits within 3.89 days around the star. This second planet in the system seems also to be a "super-Earth" with a mass of about $8.4 M_{\text {Earth }}$.
From the densities of these planets it seems that both bodies are made of rocks or water and rocks similar to our Earth. Due to the close distance of CoRoT-7b to its host star the surface temperatures can reach about $2000 \mathrm{~K}$, which indicates that the planet's environment may resample more Mercury instead of the Earth-it is most likely a tidal-locked Mercury-type planet with the density of the Earth. This would mean that the silicate surface consists of a lava lake or ocean on the planets dayside and the planets' "atmosphere-exosphere" may consist of evaporated silicates. Due to tidal locking the backside of CoRoT-7b should be very cold, so that water could be liquid in some areas or be present on its surface in form of ice (Dvorak et al., 2010). With these measurements for CoRoT-7b it has been proven that the observatory is able to discover transiting exoplanets with sizes less than 2 Earth-radii (Dvorak et al., 2010).

CoRoT-8b (Bordé et al., 2010): CoRoT-8b is a dense Saturn-class exoplanet which orbits around a K1-type star in about 6.2 days. This orbit location corresponds to a circular orbit with a semi-major axis of about $0.063 \mathrm{AU}$. The planet has a radius of $0.57 \pm 0.02$ $R_{\mathrm{Jup}}$, a mass of $0.22 M_{\mathrm{Jup}}$, resulting in a mean density of about $1.6 \mathrm{~g} \mathrm{~cm}^{-3}$. With $67 \%$ of the size of Saturn and $72 \%$ of its mass, CoRoT-8b density resembles a density comparable to that of Neptune $\left(1.76 \mathrm{~g} \mathrm{~cm}^{-3}\right)$.

CoRoT-9b (Deeg et al., 2010): is a transiting exoplanet which orbits at a relatively large distance from its G3V-type star, similar to the orbit of Mercury around the Sun. For CoRoT-9b planetary evolution models can be applied without corrections required by the influence of the parent star, as has been the case with other transiting planets. By applying planetary formation modeling one finds that the planet is mostly made of hydrogen and helium but may also contain up to 20 Earth masses of other elements, including highly pressured and very hot water and rocks (Deeg et al., 2010). It is thus very similar to the Solar System's giant planets, Jupiter and Saturn. The temperature of its 
gaseous layer is expected to be between 150 degrees and minus twenty degrees Centigrade, with minimal variations between day and night and depending on the possible presence of a layer of highly reflective clouds. The international team of astronomers participating in the discovery notes that temperate gas giants are, so far, the largest known group of planets.

Besides the nine exoplanets discussed in this short review (see planetary parameters in table), 3 more CoRoT-planets were recently confirmed and articles are currently prepared for CoRot-10b, CoRoT-11b and CoRoT-12b. Currently more candidates are discovered by careful light curve analysis. The most probable exoplanet candidate which is identified not to be a star spot or double star will move forward for groundbased RV follow-up observations.

\section{PRELIMINARY CHARACTERIZATION OF THE COROT-EXOPLANETS}

Knowing the size and mass and density of exoplanets allows some preliminary characterization. In case of hydrogen-rich gas giants whose upper atmospheres are strongly affected by the X-ray and EUV radiation as well as by the dense plasma environment of their host stars thermal evaporation and related mass loss can be estimated. In a recent study by Lammer et al. (2009) the thermal escape of hydrogen atoms from 57 transiting exoplanets was estimated by applying a modified energy-limited mass loss equation which includes Roche Lobe effects (Erkaev et al., 2007) and can reproduce the full hydrodynamic approach of studies such as that of Penz et al. (2009). By applying the same method to the exoplanets discovered by CoRoT and integrated over the age of their host stars one obtains the thermal mass loss values in percent of their mass shown in table.

By considering an uncertainty range in the hydrogen heating efficiency $\eta$ of 10 to $25 \%$, which corresponds to the ratio of the net local gas heating rate to the rate of stellar radiative energy absorption, one obtains for the low density gas giants CoRoT-1b and CoRoT-5b lost during their life-time about 1.3 and $1.56 \%(\eta=10 \%)$ or 3.07 and $3.75 \%(\eta=25 \%)$ of their initial masses. The thermal mass loss of CoRoT2b, CoRoT-3b, CoRoT-4b and CoRoT-6b and CoRoT-8b are lower and therefore negligible. As it was shown in Lammer et al. (2010) the stronger mass loss effect for CoRoT- $1 b$ and CoRoT-5b are related to the lower planetary density, closer orbital distance and stellar parameters so that the Roche lobe becomes an important factor in the mass loss evolution of these two bodies.

As shown by Lammer et al. (2009) due to the Roche lobe effect the mass loss from close-in gas giants can be very efficient at orbital distances $\sim 0.02 \mathrm{AU}$. As discussed above and shown in table most of the so far discovered transiting CoRoT-exoplanets experienced negligible mass loss during their lifetime. Compared to gas giants with high density, those with lower density are affected stronger by the thermal mass loss. Thus, the mass loss enhancing effect of the Roche lobe together with low planetary densities seems to be the main factors for high thermal mass loss rates. Depending on the heating efficiency, low density Neptune-class objects can lose their hydrogen envelopes at orbital distances $\sim 0.02$ AU (Lammer et al., 2009).

Leitzinger et al. (2009) studied thermal mass loss calculations over evolutionary time scales for the investigation if CoRoT-7b could be a remnant of an initially more massive hydrogen-rich gas giant or a hot Uranus-type exoplanet. We apply a thermal mass loss formula which yields results that are comparable to hydrodynamic loss models. The findings of these authors indicate that hydrogen-rich gas giants within the mass domain of Saturn or Jupiter cannot lose such an amount of mass that CoRoT-7b would remain as a rocky residue. Their calculations show also that the present time mass of CoRoT-7b should not be a result of evaporation of a hydrogen envelope of "hot Uranus"-type objects during $2 \mathrm{Gyr}$.

However, the non-gaseous planet CoRoT-7b can be considered as a special case. Due to the extreme environment caused by the closeness of the planets host star CoRoT-7b's initial atmosphere has most likely escaped by thermal and non-thermal loss processes (Lammer et al., 2009; Leitzinger et al., 2009). CoRoT-7b releases most likely its surface elements (silicates) on the substellar side via thermal release and stellar wind plasma sputtering. Schaefer and Fegley (2009) applied the MAGMA code of Fegley and Cameron (1987) to model the composition of silicate atmospheres which are produced by the vaporization of volatile-free "Earth composition-like" superEarth's. By considering the elements $\mathrm{Na}, \mathrm{K}, \mathrm{Fe}, \mathrm{Mg}$, $\mathrm{Si}, \mathrm{Ti}, \mathrm{Ca}, \mathrm{Al}$, and $\mathrm{O}$ and their compounds. The model calculations indicate volatile elements such as $\mathrm{H}, \mathrm{C}$, $\mathrm{N}, \mathrm{S}$, and $\mathrm{Cl}$ have been lost from the planet, but find that the silicate atmospheres which are composed primarily of $\mathrm{Na}, \mathrm{O}_{2}, \mathrm{O}, \mathrm{Mg}$ and $\mathrm{SiO}$ gas could sustain (Schaefer and Fegley, 2009). According to their study the major compounds which remain in the planetary environment are most likely $\mathrm{O}, \mathrm{Mg}$ and $\mathrm{SiO}$. The atmospheric composition may be altered by fractional vaporization, cloud condensation, photo ionization, and reaction with any residual volatile elements remaining in the atmosphere.

Recently Mura et al. (2010) applied a plasma interaction model, which is also used for the study of Mercury's solar wind plasma interaction, to the extreme stellar wind and radiation conditions which are expected at CoRoT-7b's orbit location. Mura et al. (2010) modeled a 3D spatial distribution of the expected neutral and ionized exospheric components by using a Monte-Carlo single-particle model which was developed for Mercuy (Mura et al., 2007). In this model they use a coordinate system where $\mathrm{x}$ is from the centre of the planet towards the star, $y$ is parallel to the 

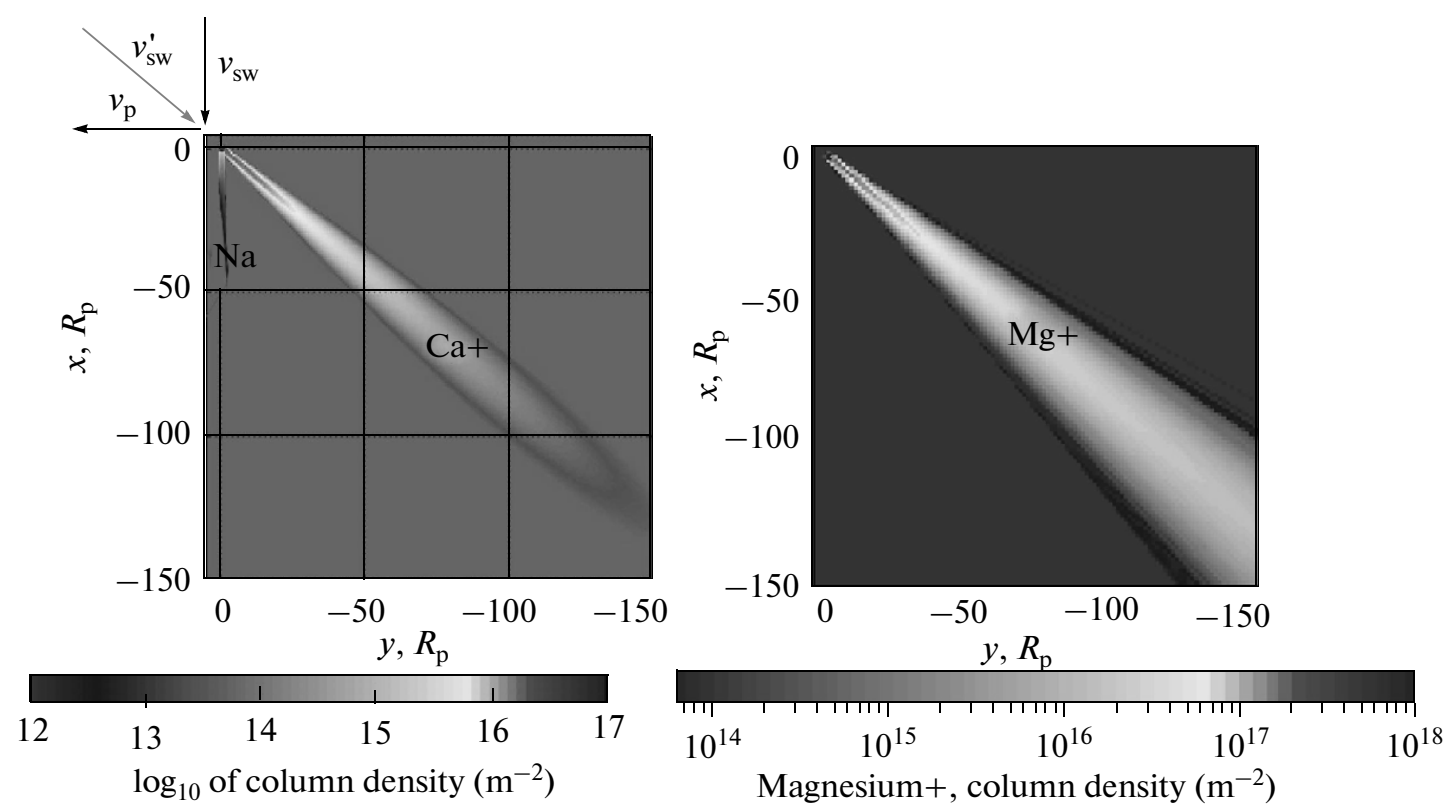

Fig. 2. Expected CoRoT-7b's stellar plasma interaction with surface evaporated $\mathrm{Na}, \mathrm{Ca}$ and $\mathrm{Mg}$. Top: Simulated $\mathrm{Na}$ and $\mathrm{Ca}{ }^{+}$column densities in the $\mathrm{x}-\mathrm{y}$ plane, integrated along the $z$ direction. The axis units are given in planetary radii. The arrows indicate the velocity of the planet/reference frame $\left(v_{\mathrm{p}}\right)$, the velocity of the stellar wind in the inertial reference frame $\left(v_{\mathrm{sw}}\right)$ and in the noninertial planetary reference frame $\left(v_{\mathrm{sw}}^{\prime}\right)$. The Na tail is narrow and short feature on the left, and is enlarged in the left panel. Bot-

tom: simulated $\mathrm{Mg}^{+}$column density in the $x-y$ plane, integrated along the $z$ direction (after Mura et al., 2010).

orbital motion, $z$ completes the right-handed reference frame. Figure 2 shows a simulation result by Mura et al. (2010) of CoRoT-7b's possible neutral Na tail and $\mathrm{Ca}^{+}$and $\mathrm{Mg}^{+}$ion tails. Depending on the surface temperature and the condensation times of $\mathrm{Ca}$ and/or $\mathrm{Mg}$, these elements could be fast ionized and may build elongated comas. Once released from the surface, elements like $\mathrm{Mg}$ are photo-ionized and trapped in the interplanetary magnetic field and are dragged away from the planet, hence forming an ion tail, which will be influenced also by a possible magnetosphere. Elements which are not so fast ionized will be heated by the stellar EUV radiation and could build up an extended exosphere/corona, which will interact with the dense stellar wind plasma flow so, that a large hydrogen ENA cloud will be produced via the charge exchange process such as around HD 209458b (Holmström et al., 2008; Eckenbäck et al., 2010).

\section{CONCLUSIONS}

The continuous observations of $10^{5}$ stars for a period of 150 days with CoRoT resulted in an efficient method to detect close-in exoplanets and even allow the discovery of rocky super-Earth-type exoplanets transiting in front of the stellar disk with a very small decrease in the flux. To obtain precise planetary parameters for the mass and the radius it is also necessary to make extensive spectroscopic measurements and also to have a good knowledge of the stellar parameters. Only after a careful and detailed analysis of the light curves together with ground-based RV follow-up observations the confirmation of a new planet can be established. Interestingly the exoplanets discovered so far by CoRoT represent different bodies with an extraordinary value for exoplanetary research. Although, most of the discovered exoplanets are gas giants with radii between $0.97 \leq R_{\mathrm{Jup}} \leq 1.49$, they have special attributes compared to many exoplanets observed by other instruments or research projects. The largest CoRoT-planets CoRoT-1b and CoRoT$4 \mathrm{~b}$ belong to the closest discovered exoplanets with very short periods of about 1.51 and 1.74 days, respectively. Three of five hot Jupiter's detected by CoRoT orbit very metal poor host stars, which is in contradiction to the usual metallicity relations found by RV searches. CoRoT-9b with orbiting around its host star at about $0.36 \mathrm{AU}$ is the coolest transiting gas giant so far discovered. Furthermore the domain of planetary masses covered by the CoRoT exoplanets is within a wide range, namely from the very massive CoRoT-3b with 21.6 $M_{\text {Jup }}$ to the super-Earth domain of CoRoT-7b of about $5 M_{\text {Earth }}$. Finally, we conclude that the results of our study show that the discovery of transiting exoplanets at orbital distances $\sim 0.015 \mathrm{AU}$ and groundbased follow-up mass determinations together with theoretical mass loss studies can bring reliable information on the statistics the of remaining cores of shrinked gas giants. 


\section{ACKNOWLEDGMENTS}

H. Lammer, M. Fridlund, J. Schneider and A. Mura acknowledge the International Space Science Institute (ISSI; Bern, Switzerland) and the ISSI team "Evolution of Exoplanet Atmospheres and their Characterization". H. Lammer and A. Mura also acknowledge fruitful discussions during various meetings related to the Europlanet N2 activities as well as within the N2 discipline working groups WG 4 and WG5. H. Lammer, R. Dvorak, J. Hasiba, H. Ottacher and M. Steller also thank the Austrian Ministry bm:bwk and ASA for funding the CoRoT project.

\section{REFERENCES}

Aigrain, S., Cameron Collier A., Ollivier, M., et al., Transiting Exoplanets from the CoRoT Space Mission IV. CoRoT-Exo-4b: a Transiting Planet in a 9.2 Day Synchronous Orbit, Astron. Astrophys., 2008, vol. 488, pp. L43-L46.

Alonso, R., Auvergne, M., Baglin, A., et al., Transiting Exoplanets from the CoRoT Space Mission II. CoRoT-Exo-2b: A Transiting Planet Around an Active G Star, Astron. Astrophys., 2008, vol. 482, pp. L21-L24.

Baglin, A., Aerts, C., and Guillot, T., The CoRoT Space Mission: Early Results, Astron. Astrophys., 2009, vol. 506, pp. 2-572.

Barge, P., Baglin, A., Auvergne, M., et al., Transiting Exoplanets from the CoRoT Space Mission I. CoRoTExo-1b: A Low-Density Short-Period Planet Around a G0V Star, Astron. Astrophys., 2008, vol. 482, pp. L17-L20.

Bordé, P., Bouchy, F., Deleul, M., et al., Transiting Exoplanets from the CoRoT Space Mission X. CoRoT-8b: A Small Saturn-Class Exoplanet, Astron. Astrophys, 2010 Submitted.

Deeg, H.J., Moutou, C., Erikson, A., et al., A Transiting Giant Planet with a Temperature Between $250 \mathrm{~K}$ and 430 K, Nature, 2010, vol. 464, pp. 384-387.

Deleuil, M., Deeg, H.J., Alonso, R., et al., Transiting Exoplanets from the CoRoT Space Mission VI. CoRoT-Exo-3b: The First Secure Inhabitant of the Brown-Dwarf Desert, Astron. Astrophys., 2008, vol. 491, pp. 889-897.

Dvorak, R., Schneider, J., and Lammer, H., and the CoRoT Team, CoRoT's First Seven Planets: An Overview, Astron. Astrophys., 2010.

Ekenbäck, A., Holmström, M., Wurz, P., et al., Energetic Neutral Atoms around HD 209458b: Estimations of Magnetospheric Properties, Astrophys. J., 2010, vol. 709, pp. 670-679.
Erkaev, N.V., Kulikov, Yu.N., Lammer, H., et al., Roche Lobe Effects on the Atmospheric Loss from "Hot Jupiters", Astron. Astrophys., 2007, vol. 472, pp. 329-334.

Fridlund, M., Hebrard, G., Alonso, R., et al., Transiting Exoplanets from the CoRoT Space Mission IX. CoRoT-6b: A Transiting "Hot Jupiter" Planet in an 8.9d Orbit Around a Low-Metallicity Star, Astron. Astrophys., 2010, vol. 512, p. A14.

Holmstrom, M., Ekenback, A., Selsis, F., et al., Energetic Neutral Atoms As the Explanation for the High-Velocity Hydrogen Around HD 209458b, Nature, 2008, vol. 451, pp. 970-972.

Lammer, H., Odert, P., Leitzinger, M., et al., Determining the Mass Loss Limit for Close-in Exoplanets: What Can We Learn from Transit Observations?, Astron. Astrophys., 2009, vol. 506, pp. 399-410.

Léger, A., Rouan, D., Schneider, J., et al., Transiting Exoplanets from the CoRoT Space Mission VIII. CoRoT-7b: The First Super-Earth with Measured Radius, Astron. Astrophys., 2009, vol. 506, pp. 287-302.

Leitzinger, M., Odert, P., Lammer, H., et al., Could CoRoT-7b Be a Remnant of an Evaporated Gas or Ice Giant?, Planet. Space Sci., 2010 submitted.

Moutou, C., Bruntt, H., Guillot, T., et al., Transiting Exoplanets from the CoRoT Space Mission V. CoRoT-Exo-4b: Stellar and Planetary Parameters, Astron. Astrophys., 2008, vol. 488, pp. L47-L50.

Mura, A., Milillo, A., Orsini, S., et al., Numerical and Analytical Model of Mercury's Exosphere: Dependence on Surface and External Conditions, Planet. Space Sci., 2007, vol. 55, pp. 1569-1583.

Mura, A., Wurz, P., Schneider, J., et al., Comet-Like TailFormation of Exopsheres of Hot Rocky Exoplanets: Possible Implications for CoRoT-7b, Icarus, 2010 submitted.

Penz, T., Erkaev, N.V., Kulikov, Yu.N., et al., Mass Loss from "Hot Jupiters" - Implications for CoRoT Discoveries, Part II: Long Time Thermal Atmospheric Evaporation Modeling, Planet. Space Sci., 2008, vol. 56, pp. 1260-1272.

Queloz, D., Bouchy, F., Moutou, C., et al., The CoRoT-7 Planetary System: Two Orbiting Super-Earths, Astron. Astrophys., 2009, vol. 506, pp. 303-319.

Rauer, H., Queloz, D., Csizmadia, Sz., et al., Transiting Exoplanets from the CoRoT Space Mission VII. The "Hot-Jupiter"-Type Planet CoRoT-5b, Astron. Astrophys., 2009, vol. 506, pp. 281-286.

Schaefer, L. and Fefley, B., Jr., Chemistry of Silicate Atmospheres of Evaporating Super-Earths, Astrophys. J. Lett, 2009, vol. 703, no. 2, pp. L113-L117.

Schneider, J. and Chevreton, M., The Photometric Search for Earth-Sized Extrasolar Planets by Occultation in Binary Systems, Astron. Astrophys., 1990, vol. 232, pp. 251-257. 